\title{
Subcutaneous Phaeohyphomycosis Caused by Exophiala salmonis
}

\author{
Young Ahn Yoon, M.D. ${ }^{1}$, Kyung Sun Park, M.D. ${ }^{1}$, Jang Ho Lee, M.T. ${ }^{1}$, Ki-Sun Sung, M.D. ${ }^{2}$, Chang-Seok Ki, M.D. ${ }^{1}$, \\ and Nam Yong Lee, M.D. ${ }^{1}$ \\ Departments of Laboratory Medicine and Genetics ${ }^{1}$, Orthopedic Surgery², Samsung Medical Center, Sungkyunkwan University School of Medicine, Seoul, Korea
}

We report a case of subcutaneous infection in a 55-yr-old Korean diabetic patient who presented with a cystic mass of the ankle. Black fungal colonies were observed after culturing on blood and Sabouraud dextrose agar. On microscopic observation, septated ellipsoidal or cylindrical conidia accumulating on an annellide were visualized after staining with lactophenol cotton blue. The organism was identified as Exophiala salmonis by sequencing of the ribosomal DNA internal transcribed spacer region. Phaeohyphomycosis is a heterogeneous group of mycotic infections caused by dematiaceous fungi and is commonly associated with immunocompromised patients. The most common clinical manifestations of subcutaneous lesions are abscesses or cystic masses. To the best of our knowledge, this is the first reported case in Korea of subcutaneous phaeohyphomycosis caused by $E$. salmonis that was confirmed by molecular analysis and identification of morphological characteristics. This case suggests that $E$. salmonis infections are no longer restricted to fish.

Key Words: Phaeohyphomycosis, Exophiala, Soft tissue infection, Sequence analysis, Korea

\author{
Received: June 18, 2012 \\ Revision received: July 30, 2012 \\ Accepted: September 12, 2012
}

\begin{abstract}
Corresponding author: Nam Yong Lee Department of Laboratory Medicine and Genetics, Samsung Medical Center, 81 Irwon-ro, Gangnam-gu, Seoul 135-710, Korea

Tel: +82-2-3410-2706

Fax: +82-2-3410-2719

E-mail: micro.lee@samsung.com
\end{abstract}

\begin{abstract}
@ The Korean Society for Laboratory Medicine. This is an Open Access article distributed under the terms of the Creative Commons Attribution Non-Commercial License (http://creativecommons.org/licenses/by-nc/3.0) which permits unrestricted non-commercial use, distribution, and reproduction in any medium, provided the original work is properly cited.
\end{abstract}

\section{INTRODUCTION}

Phaeohyphomycosis is a group of mycotic infections caused by dematiaceous (darkly pigmented) fungi that contain melanin in their cell walls. Currently, there are at least 57 genera and 104 species that can cause phaeohyphomycosis [1]. Among them, the major etiologic agents of phaeohyphomycosis are species of Bipolaris, Exophiala, Curvularia, Chaetomium, Phoma, ExserohiIum, and Wangiella. Clinical manifestations of phaeohyphomycosis vary from superficial and subcutaneous to systemic. Subcutaneous infections mostly occur on the limbs in the fingers, wrists, knees, or ankles [1]. There have been several reports of Exophiala salmonis infections in fresh and saltwater salmonids, and there has been 1 report of a human infection [2, 3]. Here, we report the first case of subcutaneous phaeohyphomycosis in Korea caused by E. salmonis that was confirmed based on morphologic characteristics and molecular analysis.

\section{CASE REPORT}

A 55-yr-old man was admitted to the hospital with a cystic mass and pus-like discharge from the left ankle (Fig. 1). Three months prior, he had undergone surgery for a medial malleolar fracture. The cystic mass was soft, palpable, non-tender, and 5-6 cm in diameter. Four weeks after surgery, post-operative complications, including redness and swelling around the surgical site, were observed. Although the infection site was incised and drained and antibiotics were administered at a local clinic, his symptoms did not improve.

At that time, the patient had been an ambulatory diabetes mellitus (DM) patient for $18 \mathrm{yr}$, and his fifth toe had been amputated $5 \mathrm{yr}$ prior due to DM complications of the foot. At the time of admission to our hospital, there were no distinctive symptoms, such as pain, heat sensation, burrows, or regional lymphadenopathy, except for the occasional pus-like discharge from 
the cystic mass. His temperature was $36^{\circ} \mathrm{C}$, and his other vital signs were within the normal limits. Serum C-reactive protein level ( $6.53 \mathrm{mg} / \mathrm{dL}$, reference range (RR): 0-0.3 mg/dL) and erythrocyte sedimentation rate (92 mm/hr, RR: 0-22 mm/hr) were elevated. The mass was incised and drained, and a fluid specimen was sent to the microbiology laboratory for culture on the second day of hospitalization.

There was no bacterial growth on the blood agar plates after 7 days of incubation, but several dark gray colonies, $1-3 \mathrm{~mm}$ in diameter, appeared along the streak line after incubation in 5\% $\mathrm{CO}_{2}$ at $35^{\circ} \mathrm{C}$ for 3 days (Fig. 2). These colonies were also observed after subculturing on Sabouraud dextrose agar that was incubated at $30^{\circ} \mathrm{C}$ for 14 days. The colonies grew to $21 \mathrm{~mm}$ in

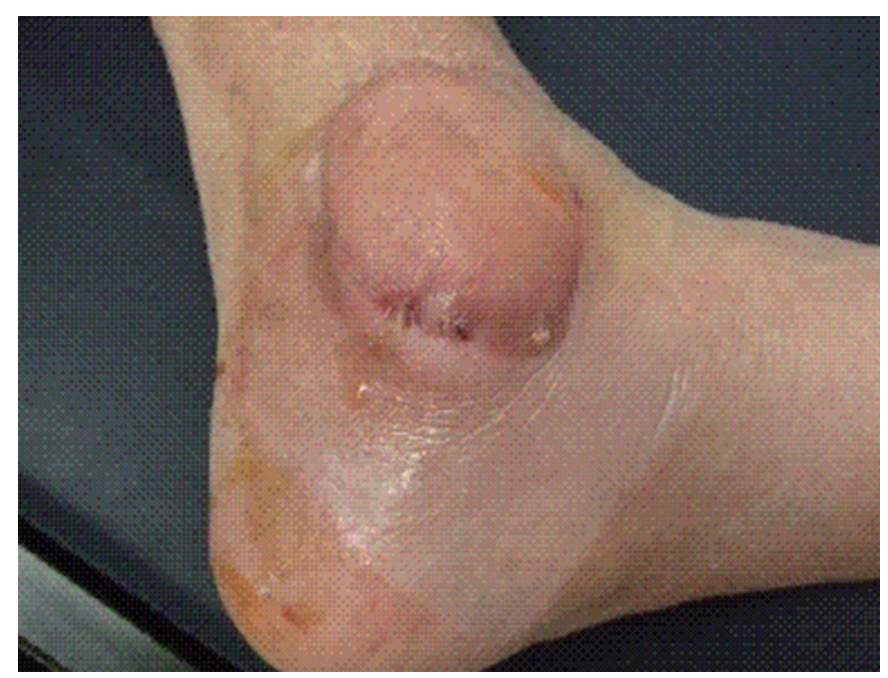

Fig. 1. Cystic mass on the left ankle. The swollen mass was soft, palpable, non-tender, and filled with pus.

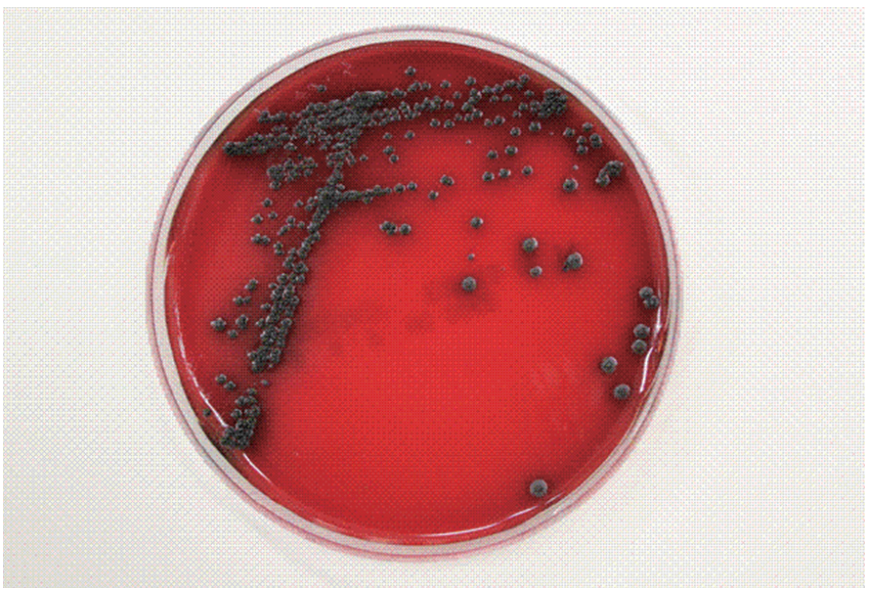

Fig. 2. Colony morphology of Exophiala salmonis on the blood agar plates after 3 days incubation. Black colonies 1-3 mm in diameter can be observed along the streak line. diameter. The colonies were circular, brownish black in color, and smooth. Initially, skin- or yeast-like colonies were observed and later developed a velvety, black felt-like appearance (Fig. 3); the reverse side of each colony was also dark. Microscopic morphologic examination using lactophenol cotton blue staining method revealed colonies consisting of septate hyphae with poorly differentiated, or sometimes tubular, short flask-shaped annellides. Septate ellipsoidal or cigar-shaped conidia accumulating at the tip of an annellide were a distinctive characteristic of this fungal colony (Fig. 4). Based on the morphologic investigation, a tentative identification of the fungi was possible, an Exophiala species.

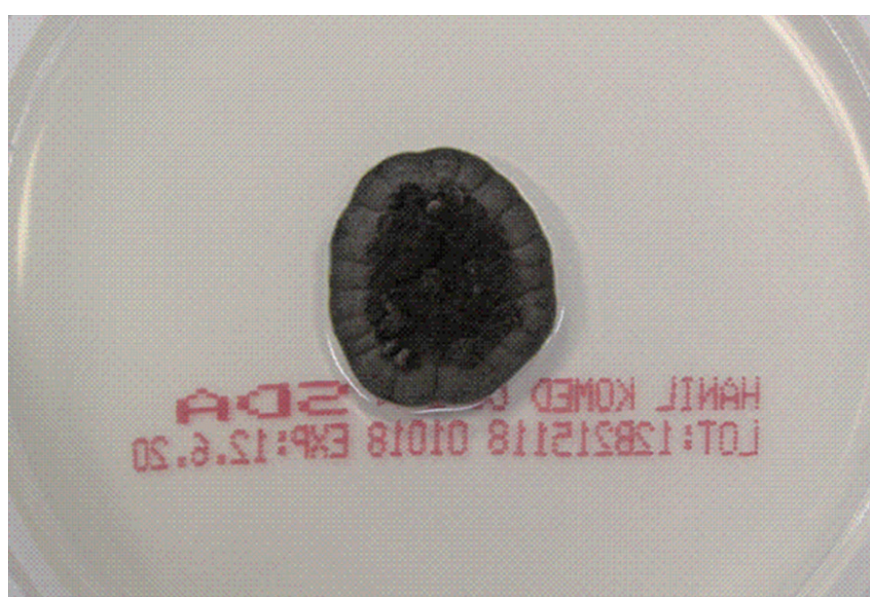

Fig. 3. Colony morphology of Exophiala salmonis on Sabouraud dextrose agar after 14 days incubation. The colony surface is brownish-black and velvety or felt-like. The reverse side of the colony is also black.

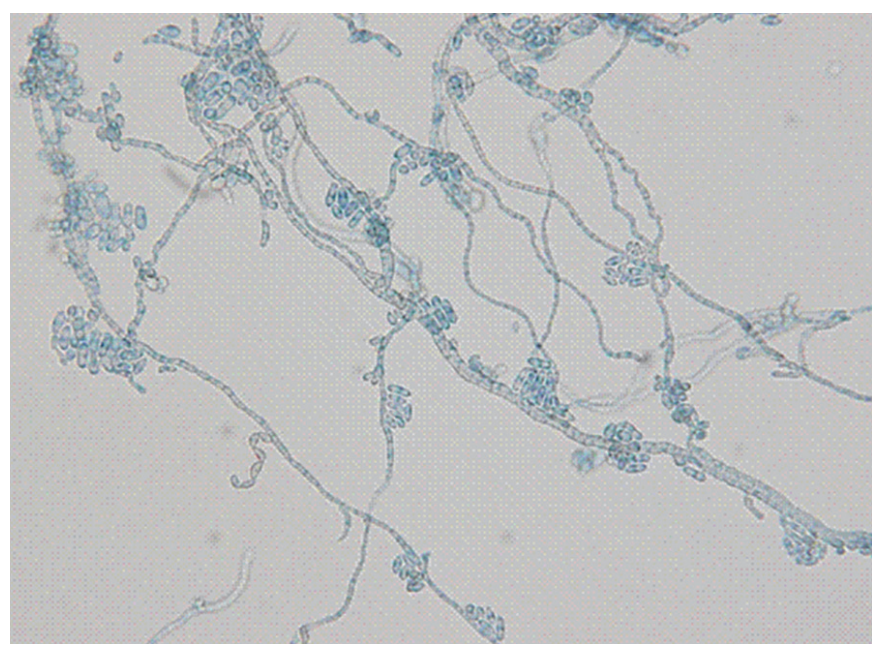

Fig. 4. Septated ellipsoidal or cylindrical conidia at the annellide. Septated hyphae with poorly differentiated or sometimes tubular, short flask-shaped annellides are observed (lactophenol cotton blue stain, $\times 400$ ). 
We performed direct sequencing analysis of the internal transcribed spacer (ITS) region of the ribosomal DNA (rDNA) for identification of the fungi upto the species level. Fungal DNA was extracted using the MagNA Pure LC DNA Isolation Kit (Roche Diagnostics, Manheim, Germany). PCR was performed using a thermal cycler (Model 9700; Applied Biosystems, Foster City, CA, USA), and amplified products were sequenced using a BigDye Terminator Cycle Sequencing Ready Reaction Kit (Applied Biosystems, Foster City, CA, USA) and an ABI Prism 3130 Genetic Analyzer (Applied Biosystems). ITS region sequences covering ITS1, the 5.8S rDNA, and ITS2 were amplified using ITS-1 (1F: 5'-TCC GTA GGT GAA CCT GCG G-3')/ITS-4 (4R: 5'TCC TCC GCT TAT TGA TAT GC-3') and ITS-5 (5F: 5'-GGA AGT AAA AGT CGT AAC AAG G -3')/ITS-4 (4R: 5'-TCC TCC GCT TAT TGA TAT GC-3') primer sets. The amplified sequences were compared and searched for in the GenBank National Center for Biotechnology Information (NCBI) database using the basic local alignment search tool (BLAST) algorithm. The GenBank BLAST search revealed 99.7\% (615/617 bp) homology with a previously sequenced $E$. salmonis strain (GenBank accession number: AY213652.1), based on the ITS rDNA gene sequencing. The second best matched strain was an Ascomycota spp. (GenBank accession number: GUg66589.1) with 94\% (602/639 bp) homology. Until discharge on day 14, the patient received surgical treatments, including incision, drainage, irrigation, and debridement and wound closure without the administration of antifungal agents and was monitored closely as an outpatient.

\section{DISCUSSION}

Phaeohyphomycosis, first described by Ajello et al. in 1974, literally means a condition of dark hyphal fungus regardless of the causative organism [4]. Phaeohyphomycosis is broadly classified as a deep systemic, subcutaneous, cutaneous, corneal, or superficial mycosis on the basis of the infection site. Subcutaneous infections occur primarily on the extremities, such as the fingers, wrists, knees, or ankles. This may be because these sites are easily exposed due to trauma and wounds and are contaminated by soil, plants, or decomposing wood material, which leads to direct fungal inoculation. Initial manifestations of subcutaneous lesions are often swollen cysts, which are filled with pus and granuloma at the site of probable trauma. These lesions should be differentiated from fibroma, lipoma, sebaceous cysts, and other disorders [1]. On histopathologic examination, an abscess surrounded by connective tissue and suppurative granulomatous inflammation was observed [5]. As the number of immunocompromised patients (solid-organ transplant recipients, patients with hematologic malignancies, and patients receiving corticosteroids) has increased, so has the number of phaeohyphomycosis reports $[6,7]$. However, the immunocompromised state is not a necessary condition for phaeohyphomycosis. Phaeohyphomycosis has also been reported in healthy patients, patients who have never received immunosuppressive drug treatment, and those testing negative for DM and HIV infections [8].

The most common agents of subcutaneous phaeohyphomycosis are Exophiala jeanselmei followed by Exophiala dermatitidis $[1,9,10]$. Although rarely seen in Korea, there have been several reports of subcutaneous phaeohyphomycosis caused by E. jeanselmei, E. dermatitis, Drechslera dematioidea, and Phoma spp. However, there are no reports of $E$. salmonis [5, 10-14] causing phaeohyphomycosis. E. salmonis is an aquatic fungus and is a widely recognized fish pathogen [3]. In 2006, Madan et al. [2] reported the first and only case of phaeohyphomycosis caused by $E$. salmonis presenting as multiple painless nodules over the elbow and knee. A yellow pus-like discharge was occasionally observed, and the patient had previously received immunosuppressive treatment with cyclophosphamide and fludarabine for non-Hodgkin's lymphoma and prednisolone for autoimmune hemolytic anemia. The etiologic agent was identified by morphological characteristics alone, as molecular diagnosis was not conducted at that time. The probable cause of inoculation of the etiologic organism was wound exposure to contaminated plant materials. In many reported cases, fungal infections were preceded by trauma to the skin. In the case presented here, the patient had a history of traumatic malleolar fracture, was suffering for DM for $18 \mathrm{yr}$, and had severe DM complications. Therefore, it was assumed that the complexity of this infection was due to trauma history, wound contamination, and delayed healing due to DM.

In recent years, diagnostic molecular techniques have been used in bacteriology and mycology. For molecular diagnosis, we used direct sequencing of ITS regions that have been used as reference sequences and accumulated in a public database. Despite the variety of resolutions available depending on the genus, sequencing of rDNA ITS regions may be useful for fungal identification as recommended by the CLSI $[15,16]$. Using this method, the isolated colonies were identified as E. salmonis when compared to the GenBank database using the BLAST algorithm. The microscopic morphology among Exophiala spp. was similar in that the young cultures were comprised of many yeast-like, budding, oval to round cells ultimately producing 
septate hyphae with numerous annellides. In addition, ellipsoidal and cylindrical conidia accumulated at the end of the conidiogenous cells. Although septate conidia with rounded distal ends are characteristic feature of $E$. salmonis, conidia without septation is sometimes observed. Due to the limitations of morphological discrimination, identification at the species level can be made via molecular analysis. A clinically accurate identification of Exophiala spp. via direct sequencing was needed, because earlier reports indicate that the clinical course differed according to species. Further, E. salmonis infections are no longer confined to fish and are now known to occur in humans [2, 17]. E. dermatitis often causes systemic infection, while E. xenobiotica and $E$. jeanselmei usually cause mild cutaneous and subcutaneous infections in humans [18].

Surgical excision of the lesion with or without the use of antifungal agents has been widely used in the treatment of subcutaneous phaeohyphomycosis. It has been reported that itraconazole, ketoconazole, amphotericin B, and flucytosine appear to be effective treatments for patients with phaeohyphomycosis [19]; however, there is no sufficient clinical data regarding the treatment of subcutaneous phaeohyphomycosis caused by $E$. salmonis. Additional research needs to be conducted in order to standardize antifungal agent regimens.

\section{Authors' Disclosures of Potential Conflicts of Interest}

No potential conflicts of interest relevant to this article were reported.

\section{REFERENCES}

1. Rinaldi MG. Phaeohyphomycosis. Dermatol Clin 1996;14:147-53.

2. Madan V, Bisset D, Harris P, Howard S, Beck MH. Phaeohyphomycosis caused by Exophiala salmonis. Br J Dermatol 2006;155:1082-4.

3. Otis EJ, Wolke RE, Blazer VS. Infection of Exophiala salmonis in Atlantic salmon (Salmo salar L.). J Wildl Dis 1985;21:61-4.

4. Ajello L, Georg LK, Steigbigel RT, Wang CJ. A case of phaeohyphomycosis caused by a new species of Phialophora. Mycologia 1974;66:490-8.

5. Suh MK, Kwon SW, Kim TH, Sun YW, Lim JW, Ha GY, et al. A case of subcutaneous phaeohyphomycosis caused by Exophiala jeanselmei. Korean J Dermatol 2005;43:124-7.

6. Koga T, Matsuda T, Matsumoto T, Furue M. Therapeutic approaches to subcutaneous mycoses. Am J Clin Dermatol 2003;4:537-43.

7. Ben-Ami R, Lewis RE, Raad II, Kontoyiannis DP. Phaeohyphomycosis in a tertiary care cancer center. Clin Infect Dis 2009;48:1033-41.

8. Rossetto AL, Dellatorre G, Pérsio RA, Romeiro JC, Cruz RC. Subcutaneous phaeohyphomycosis on the scrotum caused by Exophiala jeanselmei: case report. An Bras Dermatol 2010;85:517-20.

9. Ronan SG, Uzoaru I, Nadimpalli V, Guitart J, Manaligod JR. Primary cutaneous phaeohyphomycosis: report of seven cases. J Cutan Pathol 1993;20:223-8.

10. Suh MK. Phaeohyphomycosis in Korea. Nihon Ishinkin Gakkai Zasshi 2005;46:67-70.

11. Choi J, Lee Y, Chung HS, Koo JS, Yong D, Kim YS, et al. Subcutaneous phaeohyphomycosis caused by Phaeoacremonium species in a kidney transplant patient: the first case in Korea. Korean J Lab Med 2011;31: 201-4.

12. Jeong ES, Shin JH, Shin MG, Suh SP, Ryang DW. Fungemia due to Exophiala dermatitidis. Korean J Clin Microbiol 2010;13:135-9.

13. Kim DS, Yoon YM, Kim SW. Phaeohyphomycosis due to Exophiala dermatitidis successfully treated with Itraconazole. Korean J Med Mycol 1999;4:79-83.

14. Chang CL, Kim DS, Park DJ, Kim HJ, Lee CH, Shin JH. Acute cerebral phaeohyphomycosis due to Wangiella dermatitidis accompanied by cerebrospinal fluid eosinophilia. J Clin Microbiol 2000;38:1965-6.

15. Clinical and Laboratory Standards Institute. Interpretive criteria for identification of bacteria and fungi by DNA target sequencing. 1st ed. approved guideline, MM18-A. Wayne, PA: Clinical and Laboratory Standards Institute, 2008

16. Jang JH, Lee JH, Ki CS, Lee NY. Identification of clinical mold isolates by sequence analysis of the internal transcribed spacer region, ribosomal large-subunit D1/D2, and beta-tubulin. Ann Lab Med 2012;32: 126-32.

17. Nomura M, Maeda M, Seishima M. Subcutaneous phaeohyphomycosis caused by Exophiala jeanselmei in collagen disease patient. J Dermatol 2010;37:1046-50.

18. Aoyama Y, Nomura M, Yamanaka S, Ogawa Y, Kitajima Y. Subcutaneous phaeohyphomycosis caused by Exophiala xenobiotica in a nonHodgkin lymphoma patient. Med Mycol 2009;47:95-9.

19. Sharkey PK, Graybill JR, Rinaldi MG, Stevens DA, Tucker RM, Peterie JD, et al. Itraconazole treatment of phaeohyphomycosis. J Am Acad Dermatol 1990;23:577-86. 